\title{
A measurement of the tau lifetime
}

\section{DELPHI Collaboration}

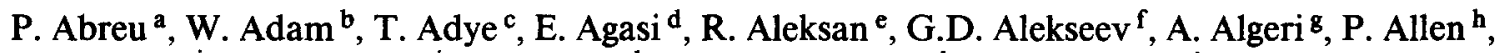
S. Almehed ${ }^{i}$, S.J. Alvsvaag ${ }^{j}$, U. Amaldi ${ }^{b}$, E.G. Anassontzis ${ }^{k}$, A. Andreazza ${ }^{\ell}$, P. Antilogus ${ }^{m}$, W.-D. Apel ${ }^{\text {n }}$, R.J. Apsimon c, Y. Arnoud ${ }^{\text {e }}$, B. Åsman ${ }^{\text {o }}$, J.-E. Augustin ${ }^{\text {p }}$, A. Augustinus d, P. Baillon ${ }^{\text {b }}$, P. Bambade ${ }^{\text {p }}$, F. Barao ${ }^{\text {a }}$, R. Barate ${ }^{q}$, G. Barbiellini ${ }^{r}$, D.Y. Bardin ${ }^{f}$, G.J. Barker ${ }^{\text {, }}$, A. Baroncelli ${ }^{t}$, O. Barring ${ }^{b}$, J.A. Barrio ${ }^{u}$, W. Bartl ${ }^{v}$, M.J. Bates ${ }^{\text {c }}$, M. Battaglia g, M. Baubillier ${ }^{\text {w }}$, K.-H. Becks x, C.J. Beeston s, M. Begalli ${ }^{y}$, P. Beilliere ${ }^{z}$, Yu. Belokopytov ${ }^{\text {aa }}$, P. Beltran ${ }^{\text {ab }}$, D. Benedic ${ }^{\text {ac }}$, A.C. Benvenuti ${ }^{\text {ad }}$, M. Berggren ${ }^{p}$, D. Bertrand ${ }^{\text {ae }}$, F. Bianchi af ${ }^{\text {, M.S. Bilenky }}{ }^{f}$, P. Billoir ${ }^{\text {w }}$, J. Bjarne ${ }^{\mathrm{i}}$, D. Bloch ${ }^{\text {ac }}$, S. Blyth ${ }^{\text {s }}$, V. Bocci ${ }^{\text {ag }}$, P.N. Bogolubov ${ }^{\text {f }}$, T. Bolognese ${ }^{\mathrm{e}}$, M. Bonesini ${ }^{\ell}$, W. Bonivento ${ }^{\ell}$, P.S.L. Booth ${ }^{\text {ah }}$, G. Borisov ${ }^{\text {aa }}$, H. Borner $^{\text {b }}$, C. Bosio ${ }^{t}$, B. Bostjancic ai, S. Bosworth ${ }^{\text {s }}$, O. Botner ${ }^{\text {aj }}$, B. Bouquet ${ }^{\text {p }}$, C. Bourdarios ${ }^{\text {p }}$, T.J.V. Bowcock ${ }^{\text {ah }}$, M. Bozzo ${ }^{\text {ak }}$, S. Braibant ${ }^{\text {ae }}$, P. Branchini ${ }^{t}$, K.D. Brand ${ }^{a \ell}$, R.A. Brenner ${ }^{\text {b }}$, H. Briand ${ }^{\text {w }}$, C. Bricman $^{\text {ae }}$, R.C.A. Brown ${ }^{\text {b }}$, N. Brummer ${ }^{\text {d }}$, J.-M. Brunet ${ }^{2}$, L. Bugge $^{\text {am }}$, T. Buran $^{\text {am }}$, H. Burmeister ${ }^{\text {b }}$, J.A.M.A. Buytaert ${ }^{\text {b }}$, M. Caccia ${ }^{\text {b }}$, M. Calvi ${ }^{\ell}$, A.J. Camacho Rozas ${ }^{\text {an }}$, R. Campion ${ }^{\text {ah }}$, T. Camporesi ${ }^{\text {b }}$, V. Canale ${ }^{\text {ag }}$, F. Cao ${ }^{\text {ae }}$, F. Carena ${ }^{\text {b }}$, L. Carroll $^{\text {ah }}$, M.V. Castillo Gimenez ${ }^{\text {h }}$, A. Cattai ${ }^{\text {b }}$, F.R. Cavallo ${ }^{\text {ad }}$, L. Cerrito ${ }^{\text {ag, }}$, V. Chabaud ${ }^{\text {b }}$, A. Chan ${ }^{\text {ao }}$, Ph. Charpentier ${ }^{b}$, L. Chaussard $^{\text {p }}$, J. Chauveau $^{\text {w }}$, P. Checchia ${ }^{\text {a }}$, G.A. Chelkov ${ }^{\mathrm{f}}$, L. Chevalier $^{\mathrm{e}}$, P. Chliapnikov ${ }^{\text {aa }}$, V. Chorowicz ${ }^{\text {w }}$, J.T.M. Chrin ${ }^{\text {h }}$, M.P. Clara af ${ }^{\text {, P. Collins }}{ }^{\text {s }}$, J.L. Contreras ${ }^{\text {, }}$,

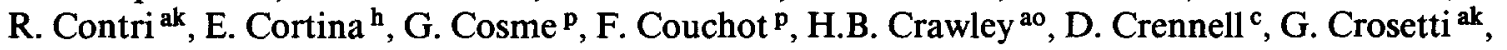
M. Crozon ${ }^{\text {z }}$, J. Cuevas Maestro ${ }^{\text {an }}$, S. Czellar ${ }^{\text {g }}$, E. Dahl-Jensen ${ }^{\text {ap }}$, B. Dalmagne ${ }^{\text {p }}$, M. Dam ${ }^{\text {am }}$, G. Damgaard ${ }^{\text {ap }}$, G. Darbo ${ }^{\text {ak }}$, E. Daubie ${ }^{\text {ae }}$, A. Daum ${ }^{\text {n }}$, P.D. Dauncey ${ }^{\text {s }}$, M. Davenport ${ }^{\text {b }}$, P. David ${ }^{\text {w }}$, J. Davies ${ }^{\text {ah }}$, W. Da Silva ${ }^{\text {w }}$, C. Defoix ${ }^{z}$, P. Delpierre ${ }^{\text {z }}$, N. Demaria ${ }^{\text {af }}$, A. De Angelis ${ }^{\mathrm{r}}$, H. De Boeck ${ }^{\mathrm{ae}}$, W. De Boer ${ }^{\mathrm{n}}$, C. De Clercq ${ }^{\text {ae }}$, M.D.M. De Fez Laso ${ }^{\mathrm{h}}$, N. De Groot ${ }^{d}$, C. De La Vaissiere ${ }^{w}$, B. De Lotto ${ }^{r}$, A. De Min ${ }^{\ell}$, H. Dijkstra ${ }^{b}$, L. Di Ciaccio ${ }^{\text {ag }}$, F. Djama ${ }^{a c}$, J. Dolbeau ${ }^{z}$, M. Donszelmann ${ }^{b}$, K. Doroba ${ }^{a q}$, M. Dracos ${ }^{b}$, J. Drees ${ }^{x}$, M. Dris ${ }^{\text {ar }}$, Y. Dufour ${ }^{b}$, F. Dupont ${ }^{\text {q }}$, L.-O. Eek ${ }^{\text {aj }}$, P.A.-M. Eerola ${ }^{\text {b }}$, R. Ehret $^{n}$, T. Ekelof ${ }^{\text {aj }}$, G. Ekspong ${ }^{\circ}$, A. Elliot Peisert ${ }^{a \ell}$, J.-P. Engel ${ }^{\text {ac }}$, N. Ershaidat ${ }^{\text {w }}$, D. Fassouliotis ${ }^{\text {ar }}$, M. Feindt ${ }^{b}$, A. Fenyuk ${ }^{\text {aa }}$, M. Fernandez Alonso ${ }^{\text {an }}$, A. Ferrer ${ }^{\text {b }}$, T.A. Filippas ${ }^{\text {ar }}$, A. Firestone ${ }^{\text {ao }}$, H. Foeth ${ }^{b}$, E. Fokitis ${ }^{\text {ar }}$, F. Fontanelli ${ }^{\text {ak }}$, K.A.J. Forbes ${ }^{\text {ah }}$, J.-L. Fousset ${ }^{\text {as }}$, S. Francon ${ }^{\mathrm{m}}$, B. Franek ${ }^{\mathrm{c}}$, P. Frenkiel ${ }^{\mathrm{z}}$, D.C. Fries ${ }^{n}$, A.G. Frodesen ${ }^{j}$, R. Fruhwirth ${ }^{\mathrm{v}}$, F. Fulda-Quenzer ${ }^{p}$, K. Furnival ${ }^{\text {ab }}$, H. Furstenau ${ }^{n}$, J. Fuster ${ }^{\text {b }}$, D. Gamba ${ }^{\text {af }}$, C. Garcia ${ }^{\text {h }}$, J. Garcia $^{\text {an }}$, C. Gaspar ${ }^{\text {b }}$, U. Gasparini ${ }^{\text {al }}$, Ph. Gavillet ${ }^{\text {b }}$, E.N. Gazis ${ }^{\text {ar }}$, J.-P. Gerber ${ }^{\text {ac }}$, P. Giacomelli ${ }^{\text {b }}$, R. Gokieli ${ }^{\text {aq }}$, B. Golob ${ }^{\text {ai }}$, V.M. Golovatyuk ${ }^{\text {, }}$, J.J. Gomez Y Cadenas ${ }^{b}$, A. Goobar ${ }^{\circ}$, G. Gopal ${ }^{\text {c }}$, M. Gorski ${ }^{\text {aq }}$, V. Gracco ${ }^{\text {ak }}$, A. Grant ${ }^{b}$, F. Grard ${ }^{\text {ae }}$, E. Graziani ${ }^{\text {t }}$, G. Grosdidier ${ }^{\text {p }}$, E. Gross ${ }^{\text {b }}$, P. Grosse-Wiesmann ${ }^{\text {b }}$, B. Grossetete ${ }^{\text {w }}$, S. Gumenyuk ${ }^{\text {aa }}$, J. Guy ${ }^{\text {c }}$, U. Haedinger ${ }^{\text {n }}$, F. Hahn ${ }^{\text {x }}$, M. Hahn ${ }^{\text {n }}$, S. Haider ${ }^{\text {d }}$, Z. Hajduk ${ }^{\text {at }}$, A. Hakansson ${ }^{\text {i }}$, A. Hallgren ${ }^{\text {aj }}$, K. Hamacher ${ }^{x}$, G. Hamel De Monchenault ${ }^{e}$, W. Hao ${ }^{\text {, }}$, F.J. Harris s, V. Hedberg ${ }^{i}$, T. Henkes ${ }^{b}$, J.J. Hernandez ${ }^{\text {h }}$, P. Herquet ${ }^{\text {ae }}$, H. Herr $^{b}$, T.L. Hessing ${ }^{\text {ah }}$, I. Hietanen ${ }^{\text {g }}$, C.O. Higgins ${ }^{\text {ah }}$, E. Higon ${ }^{\text {h }}$, H.J. Hilke ${ }^{\text {, }}$, S.D. Hodgson ${ }^{\text {s }}$, T. Hofmokl ${ }^{\text {aq }}$, R. Holmes ${ }^{\text {ao }}$, S.-O. Holmgren ${ }^{\circ}$, D. Holthuizen ${ }^{\text {d, P.F. Honore }}{ }^{z}$, J.E. Hooper ${ }^{\text {ap }}$, M. Houlden ${ }^{\text {ah }}$, J. Hrubec $^{\text {v }}$, K. Huet ${ }^{\text {ae }}$, P.O. Hulth ${ }^{\circ}$, K. Hultqvist ${ }^{\circ}$, P. Ioannou ${ }^{k}$, P.-S. Iversen ${ }^{j}$, J.N. Jackson ${ }^{\text {ah }}$, 
P. Jalocha $^{\text {at }}$, G. Jarlskog ${ }^{i}$, P. Jarry ${ }^{\text {e }}$, B. Jean-Marie ${ }^{\text {p }}$, E.K. Johansson ${ }^{0}$, D. Johnson ${ }^{\text {ah }}$, M. Jonker ${ }^{b}$, L. Jonsson ${ }^{i}$, P. Juillot ${ }^{\text {ac }}$, G. Kalkanis ${ }^{k}$, G. Kalmus ${ }^{c}$, F. Kapusta ${ }^{w}$, M. Karlsson ${ }^{b}$, E. Karvelas ${ }^{\text {ab }}$, S. Katsanevas ${ }^{k}$, E.C. Katsoufis ${ }^{a r}$, R. Keranen ${ }^{b}$, J. Kesteman ${ }^{a c}$, B.A. Khomenko ${ }^{f}$, N.N. Khovanski ${ }^{f}$, B. King ${ }^{\text {ah }}$, N.J. Kjaer ${ }^{b}$, H. Klein ${ }^{b}$, A. Klovning ${ }^{j}$, P. Kluit ${ }^{d}$, A. Koch-Mehrin ${ }^{\text {, }}$, J.H. Koehne ${ }^{n}$, B. Koene ${ }^{d}$, P. Kokkinias ${ }^{\text {ab }}$, M. Koratzinos ${ }^{\text {am }}$, K. Korcyl ${ }^{\text {at }}$, A.V. Korytov ${ }^{f}$, V. Kostioukhine ${ }^{\text {aa }}$, C. Kourkoumelis ${ }^{k}$, O. Kouznetsov ${ }^{\text {f }}$, P.H. Kramer ${ }^{\text {x }}$, J. Krolikowski aq, I. Kronkvist ${ }^{\mathrm{i}}$, U. Kruener-Marquis ${ }^{\mathrm{x}}$, W. Kucewicz ${ }^{\text {at }}$, K. Kulka ${ }^{\text {aj }}$, K. Kurvinen ${ }^{\mathrm{g}}, \mathrm{C}$. Lacasta ${ }^{\mathrm{h}}$, C. Lambropoulos ${ }^{a b}$, J.W. Lamsa ${ }^{\text {ao }}$, L. Lanceri ${ }^{r}$, V. Lapin ${ }^{\text {aa }}$, J.-P. Laugier ${ }^{\mathrm{e}}$, R. Lauhakangas ${ }^{\mathrm{g}}$, G. Leder ${ }^{v}$, F. Ledroit ${ }^{q}$, R. Leitner ${ }^{\text {au }}$, Y. Lemoigne ${ }^{e}$, J. Lemonne $^{\text {ae }}$, G. Lenzen $^{\mathrm{x}}$, V. Lepeltier ${ }^{p}$,

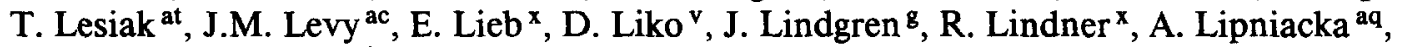
I. Lippi ${ }^{\text {al }}$, B. Loerstad ${ }^{i}$, M. Lokajicek ${ }^{\text {av }}$, J.G. Loken ${ }^{\text {s }}$, A. Lopez-Fernandez ${ }^{\text {b }}$, M.A. Lopez Aguera ${ }^{\text {an }}$, M. Los ${ }^{\text {d }}$, D. Loukas ${ }^{\text {ab }}$, J.J. Lozano ${ }^{h}$, P. Lutz ${ }^{z}$, L. Lyons ${ }^{\text {, }}$, G. Maehlum $^{\text {am }}$, J. Maillard ${ }^{\text {z }}$, A. Maio ${ }^{\text {a }}$, A. Maltezos ${ }^{\text {ab }}$, F. Mandl ${ }^{\text {v }}$, J. Marco ${ }^{\text {an }}$, M. Margoni ${ }^{\text {a }}$, J.-C. Marin ${ }^{\text {b }}$, A. Markou ${ }^{\text {ab }}$, T. Maron ${ }^{\text {x }}$, S. Marti ${ }^{\text {h }}$, L. Mathis ${ }^{\text {ao }}$, F. Matorras ${ }^{\text {an }}$, C. Matteuzzi ${ }^{\ell}$, G. Matthiae ${ }^{\text {ag }}$, M. Mazzucato ${ }^{\text {al }}$, M. Mc Cubbin ${ }^{\text {ah }}$, R. Mc Kay ${ }^{\text {ao }}$, R. Mc Nulty ${ }^{\text {ah }}$, G. Meola ${ }^{\text {ak, }}$, C. Meroni ${ }^{\ell}$, W.T. Meyer ${ }^{\text {ao }}$, M. Michelotto ${ }^{a \ell}$, I. Mikulec ${ }^{\mathrm{v}}$, L. Mirabito ${ }^{\mathrm{m}}$, W.A. Mitaroff ${ }^{\mathrm{v}}$, G.V. Mitselmakher ${ }^{f}$, U. Mjoernmark ${ }^{i}$, T. Moa ${ }^{\circ}$, R. Moeller ${ }^{\text {ap }}$, K. Moenig ${ }^{b}$, M.R. Monge ${ }^{\text {ak }}$, P. Morettini ak, H. Mueller ${ }^{n}$, W.J. Murray ${ }^{\text {c }}$, G. Myatt ${ }^{\text {s, F.L. Navarria }}{ }^{\text {ad }}$, P. Negri ${ }^{\ell}$, B.S. Nielsen $^{\text {ap }}$, B. Nijjhar ${ }^{\text {ah }}$, V. Nikolaenko ${ }^{\text {aa }}$, P.E.S. Nilsen ${ }^{j}$, P. Niss ${ }^{\circ}$, V. Obraztsov ${ }^{\text {aa }}$, A.G. Olshevski ${ }^{\text {f }}$, R. Orava ${ }^{\mathrm{g}}$, A. Ostankov ${ }^{\text {aa }}$, K. Osterberg ${ }^{\mathrm{g}}$, A. Ouraou ${ }^{\mathrm{e}}$, M. Paganoni ${ }^{\ell}$, R. Pain ${ }^{\text {w, Th.D. Papadopoulou }}{ }^{\text {ar }}$, L. Pape ${ }^{\mathrm{b}}$, F. Parodi ak, A. Passeri ${ }^{\mathrm{t}}$, M. Pegoraro ${ }^{\mathrm{a} \ell}$,

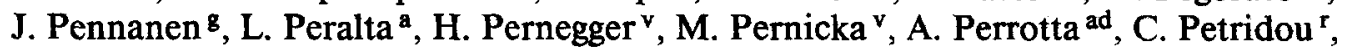
A. Petrolini ${ }^{\text {ak }}$, T.E. Pettersen ${ }^{a \ell}$, F. Pierre ${ }^{e}$, M. Pimenta ${ }^{a}$, O. Pingot ${ }^{\text {ae }}$, S. Plaszczynski ${ }^{p}$, O. Podobrin ${ }^{\text {n }}$, M.E. Pol ${ }^{\text {b }}$, G. Polok ${ }^{\text {at }}$, P. Poropat $^{\text {r }}$, P. Privitera ${ }^{\text {n }}$, A. Pullia ${ }^{\ell}$, D. Radojicic ${ }^{\text {, }}$, S. Ragazzi ${ }^{\ell}$, H. Rahmani ${ }^{\text {ar }}$, P.N. Ratoff ${ }^{\text {aw }}$, A.L. Read ${ }^{\text {am }}$, N.G. Redaelli ${ }^{\ell}$, M. Regler $^{\mathbf{v}}$, D. Reid ${ }^{\text {b }}$, P.B. Renton ${ }^{\text {s }}$, L.K. Resvanis ${ }^{\text {k }}$, F. Richard ${ }^{\text {p }}$, M. Richardson ${ }^{\text {ah }}$, J. Ridky ${ }^{\text {av }}$, G. Rinaudo af , I. Roditi ay , A. Romero af, I. Roncagliolo ${ }^{\text {ak }}$, P. Ronchese ${ }^{\text {al }}$, C. Ronnqvist ${ }^{8}$, E.I. Rosenberg ${ }^{a 0}$, S. Rossi ${ }^{b}$, U. Rossi ${ }^{\text {ad }}$, E. Rosso ${ }^{\text {b }}$, P. Roudeau ${ }^{\text {p }}$, T. Rovelli ad, W. Ruckstuhl ${ }^{d}$, V. Ruhlmann-Kleider ${ }^{\text {e }}$, A. Ruiz ${ }^{\text {an }}$, A. Rybin ${ }^{\text {aa }}$, H. Saarikko ${ }^{\text {, }}$ Y. Sacquin ${ }^{\text {e }}$, G. Sajot ${ }^{\text {q }}$, J. Salt ${ }^{\text {h }}$, J. Sanchez ${ }^{\text {, }}$ M. Sannino ${ }^{\text {ak }}$, S. Schael ${ }^{\text {b }}$, H. Schneider ${ }^{\text {n }}$, B. Schulze ${ }^{\text {ag }}$, M.A.E. Schyns ${ }^{x}$, G. Sciolla ${ }^{\text {af }}$, F. Scuri ${ }^{r}$, A.M. Segar ${ }^{\text {s }}$, A. Seitz ${ }^{\text {n }}$, R. Sekulin ${ }^{c}$, M. Sessa ${ }^{r}$, G. Sette $^{\text {ak }}$, R. Seufert ${ }^{\text {, }}$, R.C. Shellard ${ }^{y}$, I. Siccama ${ }^{d}$, P. Siegrist ${ }^{e}$, S. Simonetti ${ }^{\text {ak }}$, F. Simonetto ${ }^{\text {al }}$, A.N. Sisakian ${ }^{f}$, G. Skjevling ${ }^{\text {am }}$, G. Smadja ${ }^{\text {e,m }}$, G.R. Smith $^{c}$, R. Sosnowski ${ }^{\text {aq }}$, D. Souza-Santos ${ }^{y}$, T.S. Spassoff ${ }^{q}$, E. Spiriti ${ }^{t}$, S. Squarcia ${ }^{\text {ak }}$, H. Staeck ${ }^{x}$, C. Stanescu ${ }^{t}$, S. Stapnes ${ }^{\text {am }}$, G. Stavropoulos ${ }^{\text {ab }}$, F. Stichelbaut ${ }^{\text {ae }}$, A. Stocchi ${ }^{p}$, J. Strauss ${ }^{v}$, J. Straver ${ }^{b}$, R. Strub ${ }^{\text {ac }}$, B. Stugu ${ }^{j}$, M. Szczekowski ${ }^{b}$, M. Szeptycka ${ }^{\text {aq }}$, P. Szymanski ${ }^{\text {aq }}$, T. Tabarelli ${ }^{\ell}$, O. Tchikilev aa ${ }^{\text {aa }}$ G.E. Theodosiou ${ }^{\text {ab }}$, A. Tilquin ${ }^{\text {as }}$, J. Timmermans ${ }^{d}$, V.G. Timofeev ${ }^{f}$, L.G. Tkatchev ${ }^{f}$, T. Todorov ${ }^{a c}$, D.Z. Toet ${ }^{d}$, O. Toker ${ }^{g}$, B. Tome ${ }^{a}$, E. Torassa ${ }^{\text {af }}$, L. Tortora ${ }^{t}$, D. Treille ${ }^{b}$, U. Trevisan ${ }^{\text {ak }}$, W. Trischuk ${ }^{b}$, G. Tristram ${ }^{z}$, C. Troncon ${ }^{\ell}$, A. Tsirou ${ }^{b}$, E.N. Tsyganov ${ }^{\text {f }}$, M.-L. Turluer ${ }^{\mathrm{e}}$, T. Tuuva $^{\mathrm{g}}$, I.A. Tyapkin ${ }^{\mathrm{w}}$, M. Tyndel ${ }^{\text {c }}$, S. Tzamarias ${ }^{\text {ah }}$, S. Ueberschaer ${ }^{\mathrm{x}}$, O. Ullaland ${ }^{\mathrm{b}}$, V. Uvarov ${ }^{\text {aa }}, \mathrm{G}$. Valenti ${ }^{\text {ad }}$, E. Vallazza af, J.A. Valls Ferrer ${ }^{\text {h }}$, C. Vander Velde ${ }^{\text {ae }}$, G.W. Van Apeldoorn ${ }^{d}$, P. Van Dam ${ }^{\text {d, }}$ M. Van Der Heijden ${ }^{d}$, W.K. Van Doninck ${ }^{\text {ae }}$, P. Vaz ${ }^{\text {b }, ~ G . ~ V e g n i ~}{ }^{\ell}$, L. Ventura ${ }^{a}{ }^{\ell}$, W. Venus ${ }^{\text {c, }}$,

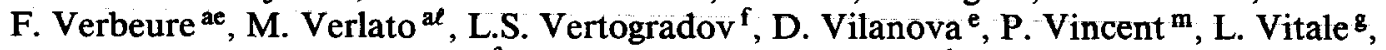
E. Vlasov $^{\text {aa }}$, A.S. Vodopyanov ${ }^{\mathrm{f}}$, M. Vollmer ${ }^{\mathrm{x}}$, G. Voulgaris $^{\mathrm{k}}$, M. Voutilainen ${ }^{\mathrm{g}}$, V. Vrba ${ }^{\mathrm{t}}$, H. Wahlen ${ }^{\mathrm{x}}$, C. Walck ${ }^{\mathrm{o}}, \mathrm{F}$. Waldner ${ }^{\mathrm{r}}, \mathrm{M}$. Wayne ${ }^{\mathrm{ao}}, \mathrm{A}$. Wehr ${ }^{\mathrm{x}}, \mathrm{M}$. Weierstall ${ }^{\mathrm{x}}$, P. Weilhammer $^{\mathrm{b}}$, J. Werner ${ }^{\mathrm{x}}$, A.M. Wetherell ${ }^{\mathrm{b}}$, J.H. Wickens ${ }^{\mathrm{ae}}$, G.R. Wilkinson ${ }^{\mathrm{s}}$, W.S.C. Williams ${ }^{\mathrm{s}}$, M. Winter ${ }^{\text {ac }}$, M. Witek ${ }^{\text {at }}$, G. Wormser ${ }^{\mathrm{p}}$, K. Woschnagg ${ }^{\text {aj }}$, N. Yamdagni ${ }^{\circ}$, P. Yepes ${ }^{\text {b }}$, 
A. Zaitsev ${ }^{\text {aa }}$, A. Zalewska ${ }^{\text {at }}$, P. Zalewski ${ }^{\text {p }}$, D. Zavrtanik ${ }^{\text {ai }}$, E. Zevgolatakos ${ }^{\text {ab }}$, G. Zhang ${ }^{\text {x }}$, N.I. Zimin ${ }^{f}$, M. Zito ${ }^{\text {e }}$, R. Zuberi ${ }^{\text {s }}$, R. Zukanovich Funchal ${ }^{z}$, G. Zumerle ${ }^{\text {al }}$ and J. Zuniga $^{\text {h }}$

a LIP, IST, FOUL, Av. Elias Garcia 14 - 1o, P-1000 Lisbon Codex, Portugal

b CERN, CH-1211 Geneva 23, Switzerland

c Rutherford Appleton Laboratory, Chilton, Didcot OXI1 0QX, UK

d NIKHEF-H, Postbus 41882, NL-1009 DB Amsterdam, The Netherlands

- Centre d'Etude de Saclay, DSM/DAPNIA, F-91191 Gif-sur-Yvette Cedex, France

f Joint Institute for Nuclear Research, Dubna, Head Post Office, P.O. Box 79, 101000 Moscow, Russian Federation

B Research Institute for High Energy Physics, University of Helsinki, Siltavuorenpenger $20 \mathrm{C}$, SF-00170 Helsinki 17, Finland

h IFIC, Valencia-CSIC, and DFAMN, Universidad de Valencia, Avda. Dr. Moliner 50, E-46100 Burjassot (Valencia), Spain

i Department of Physics, University of Lund, Sölvegatan 14, S-22363 Lund, Sweden

j Department of Physics, University of Bergen, Allégaten 55, N-5007 Bergen, Norway

k Physics Laboratory, University of Athens, Solonos Street 104, GR-10680 Athens, Greece

¿ Dipartimento di Fisica, Università di Milano and INFN, Via Celoria 16, I-20133 Milan, Italy

m Université Claude Bernard de Lyon, IPNL, IN2P3-CNRS, F-69622 Villeurbanne Cedex, France

n Institut für Experimentelle Kernphysik, Universität Karlsruhe, Pf. 6980, W-7500 Karlsruhe 1, FRG

- Institute of Physics, University of Stockholm, Vanadisvägen 9, S-113 46 Stockholm, Sweden

P Laboratoire de l'Accélérateur Linéaire, IN2P3-CNRS, Université de Paris-Sud, Bâtiment 200, F-91405 Orsay, France

q Institut des Sciences Nucléaires, Université de Grenoble 1, F-38026 Grenoble, France

' Dipartimento di Fisica, Università di Trieste and INFN, Via A. Valerio 2, I-34127 Trieste, Italy and Istituto di Fisica, Università di Udine, I-33100 Udine, Italy

s Nuclear Physics Laboratory, University of Oxford, Keble Road, Oxford OXI 3RH, UK

I Istituto Superiore di Sanità, Istituto Nazionale di Fisica Nucleare (INFN), Viale Regina Elena 299, I-00161 Rome, Italy

"Universidad Complutense, Avda. Complutense s/n, E-28040 Madrid, Spain

v Institut für Hochenergiephysik, Österreichische Akademie der Wissenschaften, Nikolsdorfergasse 18, A-1050 Vienna, Austria

w LPNHE, Universités Paris VI et VII, Tour 33 (RdC), 4 place Jussieu, F-75252 Paris Cedex 05, France

x Fachbereich Physik, University of Wuppertal, Pf. 100 127,W-5600 Wuppertal 1, FRG

y Departamento de Fisica, Pontificia Universidade Católica, C.P. 38071, RJ-22453 Rio de Janeiro, Brazil

${ }^{\mathrm{z}}$ Laboratoire de Physique Corpusculaire, IN2P3-CNRS, Collège de France, F-75231 Paris Cedex 5, France

a Institute for High Energy Physics, Serpukhov, P.O. Box 35, 142284 Protvino (Moscow Region), Russian Federation

ab Institute of Nuclear Physics, NCSR Demokritos, P.O. Box 60228, GR-15310 Athens, Greece

ac Centre de Rercherche Nucléaire, IN2P3-CNRS/ULP, B.P. 20, F-67037 Strasbourg Cedex, France

ad Dipartimento di Fisica, Università di Bologna and INFN, Via Irnerio 46, 1-40126 Bologna, Italy

ae Physics Department, Universitaire Instelling Antwerpen, Universiteitsplein $1, B-2610$ Wilrijk, Belgium and $\overline{I I H E}, U L B-V U B$, Pleinlaan 2, B-1050 Brussels, Belgium

and Faculté des Sciences, Université de l'Etat Mons, Av. Maistriau 19, B-7000 Mons, Belgium

af Dipartimento di Fisica Sperimentale, Università di Torino and INFN, Via P. Giuria 1, I-10125 Turin, Italy

as Dipartimento di Fisica, Università di Roma II and INFN, Tor Vergata, I-00173 Rome, Italy

ah Department of Physics, University of Liverpool, P.O. Box 147, Liverpool L69 3BX, UK

ai J. Stefan Institute and Department of Physics, University of Ljubljana, Jamova 39, SI-61000 Ljubljana, Slovenia

aj Department of Radiation Sciences, University of Uppsala, P.O. Box 535, S-751 21 Uppsala, Sweden

ak Dipartimento di Fisica, Università di Genova and INFN, Via Dodecaneso 33, I-16146 Genoa, Italy

al Dipartimento di Fisica, Università di Padova and INFN, Via Marzolo 8, I-35131 Padua, Italy

am Physics Department, University of Oslo, Blindern, N-1000 Oslo 3, Norway

an Facultad de Ciencias, Universidad de Santander, Av, de los Castros, E-39005 Santander, Spain

ao Ames Laboratory and Department of Physics, Iowa State University, Ames, IA 50011, USA

ap Niels Bohr Institute, Blegdamsvej 17, DK-2100 Copenhagen $\emptyset$, Denmark

aq Institute for Nuclear Studies, and University of Warsaw, Ul. Hoża 69, PL-00681 Warsaw, Poland

ar Physics Department, National Technical University, Zografou Campus, GR-15773 Athens, Greece

as Université d'Aix, Marseille II, CPP, IN2P3-CNRS, F-13288 Marseille Cedex 09, France

at High Energy Physics Laboratory, Institute of Nuclear Physics, Ul. Kawiory 26 a, PL-30055 Cracow 30, Poland

au NC, Nuclear Centre of MFF, Charles University, Areal MFF, V Holesovickach 2, 18000 Prague 8, Czech Republic 
av FZU. Institute of Physics of the CAS High Energy Physics Division, Na Slovance 2, 180 40, Prague 8, Czech Republic

aw School of Physics and Materials, University of Lancaster, Lancaster LAI 4 YB, UK

ay Centro Brasileiro de Pesquisas Fisicas, rua Xavier Sigaud 150, RJ-22290 Rio de Janeiro, Brazil

Received 4 February 1993

The tau lepton lifetime is measured using four different methods with the DELPHI detector. Three measurements using one prong decays are combined, accounting for correlations, resulting in $\tau_{\tau}=298 \pm 7$ (stat.) \pm 4 (syst.) fs while the decay length distribution of three prong decays gives $\tau_{z}=298 \pm 13$ (stat.) \pm 5 (syst.) fs. The combined result is $\tau_{\tau}=298 \pm 7 \mathrm{fs}$. The ratio of the Fermi coupling constant from tau decay relative to that from muon decay is found to be $0.985 \pm 0.013$, compatible with lepton universality.

\section{Introduction}

The tau lepton is a fundamental constituent of the standard model and its lifetime can be used to test the model predictions. In particular, lepton universality can be tested using the relationship

$\tau_{\tau}=\tau_{\mu}\left(\frac{G_{\mu}}{G_{\tau}}\right)^{2}\left(\frac{m_{\mu}}{m_{\tau}}\right)^{5} \times \mathrm{BR}\left(\tau^{-} \rightarrow e^{-\overline{\nu_{e}} \nu_{\tau}}\right)$

where $\tau_{\mu, \tau}$ and $m_{\mu, \tau}$ are the lifetimes and masses of the muon and tau respectively and $G_{\mu, \tau}$ are the Fermi constants determined from muon and tau decay [1].

The lifetime measurements presented here were derived from the data taken by the DELPHI experiment at LEP during 1991. The $\tau^{+} \tau^{-}$event selection criteria were the same as those used for the $Z \rightarrow \tau^{+} \tau^{-}$ lineshape measurement [2]. An improved three layer silicon Microvertex Detector, installed for the 1991 data taking, was used to provide the precise $r \phi \#$ charged particle measurements necessary to observe the short tau decay distance.

Four techniques were used to measure the lifetime. Three methods measured tau pairs both of which decayed into a single charged particle plus neutral particles while the fourth was used to study tau decays producing three charged particles. In the first method, the lifetime was extracted from a measurement of the distance of closest approach of the decay particle trajectory to the centre of the interaction region, referred to as the impact parameter. In the second and third methods, the correlation between impact parameter

\#1 $r, \phi$ and $z$ define a cylindrical co-ordinate system where $+z$ coincides with the electron beam direction and the origin coincides with the interaction point. and lifetime was exploited for events where both taus, in a single $Z$ event, decayed into one charged particle. The second method used the correlation between the impact parameter difference and the decay angle difference reducing the uncertainty arising from the unmeasured tau decay angles. The third method used the miss distance between tau decay tracks, defined as the sum of the impact parameters. In this case the effect of the unknown tau pair production point was greatly reduced. The fourth method reconstructed the decay vertex for taus which decayed into three charged particles detected in the Microvertex Detector. As the interaction region of the LEP beams was small compared to the mean decay length, the production point of the taus could be taken as its centre, allowing the decay length to be determined and the lifetime to be calculated.

The Monte Carlo program KORALZ [3] was used to model tau decays in all of the above analyses.

A brief description of the DELPHI tracking system is given in section 2. Section 3 describes the tau lifetime measurements made with decays producing one charged particle (the impact parameter, decay angle correlation and miss distance methods). Section 4 details the decay length analysis applied to the three prong decays. Finally, section 5 presents the combined result, accounting for correlations.

\section{The DELPHI tracking system}

The DELPHI detector is described in ref. [4]. All four analyses used the DELPHI charged particle tracking system in a $1.2 \mathrm{~T}$ solenoidal magnetic field whose axis is parallel to the beam. 
Closest to the beam axis is the Microvertex Detector (VD) which is discussed in more detail below. Outside it is the Inner Detector (ID), a gas detector with a jet-chamber geometry. It produces 24 points per track, yielding a track element with an $r \phi$ resolution of $60 \mu \mathrm{m}$. The Time Projection Chamber (TPC) is the main tracking detector of DELPHI, situated between radii of $35 \mathrm{~cm}$ and $111 \mathrm{~cm}$. Up to 16 points per track produce a track element with an $r \phi$ resolution of $200 \mu \mathrm{m}$. This tracking system has a precision in polar angle, $\theta$, of $1.7 \mathrm{mrad}$.

The DELPHI Microvertex Detector [5] used in these analyses consists of three concentric layers of silicon strip detectors at average radii of 6.3, 9.0 and $11.0 \mathrm{~cm}$, giving full azimuthal coverage in the polar angular region $43^{\circ}<\theta<137^{\circ}$. Each layer has $24 \mathrm{sec}-$ tors with a (10-15)\% overlap in $\phi$. A sector is subdivided along the beam direction into 4 silicon strip detectors. The silicon strips are parallel to the beam direction and have a pitch of $25 \mu \mathrm{m}$ with every second strip read out by capacitive pick-up. With this geometry, an intrinsic resolution in the $r \phi$ plane of $6 \mu \mathrm{m}$ is obtained using charge division. The relative position of the modules was surveyed to an accuracy of $20 \mu \mathrm{m}$ in three dimensions before installation in DEL PHI. Movement with respect to the rest of the DELPHI detector was monitored using lasers and found to be less than $5 \mu \mathrm{m}$ over the running period. The final alignment, described in ref. [5], was carried out using tracks from $\mu^{+} \mu^{-\# 2}$ decays of the $Z$, selected as described in ref. [6].

The $\mu^{+} \mu^{-}$and $e^{+} e^{-}$miss distance, the distance of closest approach of the two leptons, calculated using a full track fit to TPC, ID and VD hits on at least the inner and outermost layers had a standard deviation of $37 \pm 3 \mu \mathrm{m}$, corresponding to a track extrapolation resolution at the vertex $\sigma_{\mathrm{ext}}=37 \mu \mathrm{m} / \sqrt{2}=26 \pm 2 \mu \mathrm{m}$. The VD dominated this measurement, its single point resolution was determined to be $8 \mu \mathrm{m}$. This included an uncertainty of $6 \mu \mathrm{m}$ from the intrinsic detector resolution and $5 \mu \mathrm{m}$ from the alignment procedure.

The momentum dependent behaviour of the impact parameter resolution has been studied with lower mo-

*2 The symbol $\mu^{+} \mu^{-}$refers to muons produced in the reaction $e^{+} e^{-} \rightarrow \mu^{+} \mu^{-}$and not to tau decays producing muons. Similarly the symbol $e^{+} e^{-}$refers to electron final states from the reaction $e^{+} e^{-} \rightarrow e^{+} e^{-}$. mentum tracks in hadronic $Z$ decays [5]. This combined with the above $\mu^{+} \mu^{-}$and $e^{+} e^{-}$studies yields a track extrapolation uncertainty, $\sigma_{j}$ (in $\mu \mathrm{m}$ ), of the form:

$\sigma_{j}=\sqrt{26^{2}+\left(\frac{59}{p_{t_{j}} \sqrt{\sin \theta_{j}}}\right)^{2}}$.

The second term is a parameterisation of the multiple scattering in the $r \phi$ plane due to the beam-pipe wall and the first layer of the VD, where $p_{t}$, is the transverse momentum, in $\mathrm{GeV} / c$ of particle $j$, and $\theta_{j}$ the polar angle with respect to the beam axis.

In all of the following analyses, the tau production point was taken as the centre of the interaction region which was determined every one hundred hadronic $Z$ decays with a precision of better than $15 \mu \mathrm{m}$. Using $\mu^{+} \mu^{-}$events, it was found that the $x$ and $y$ projections of the interaction region were well represented by gaussian distributions with $\sigma_{x}=145 \mu \mathrm{m}$ and $\sigma_{y}=7 \mu \mathrm{m}$. The effects due to the size of the interaction region were accounted for by using a resolution function based on $\mu^{+} \mu^{-}$and $e^{+} e^{-}$events as described below.

\section{One prong lifetime measurements}

For these analyses, only events where both taus decayed into a single charged particle were considered. This gave a sample of 4096 tau pair events. Charged particle tracks (including the $e^{+} e^{-}$and $\mu^{+} \mu^{-}$events used to measure the resolution functions) were further required to satisfy the following criteria:

(i) at least 11 points in the TPC;

(ii) at least two layers with hits in the VD;

(iii) a $\chi^{2}$ probability for the track fit in the TPC and VD greater than 0.01 ;

(iv) the increase in the $\chi^{2}$ of the track fit when the VD points were added had to have a probability greater than 0.01 , where the number of degrees of freedom was taken as the number of hits in the VD. This cut, while strongly correlated with the previous one, ensured that all tracks in the sample had an extrapolation uncertainty consistent with the VD resolution quoted above. Both $\chi^{2}$ probability distributions were uniform; 

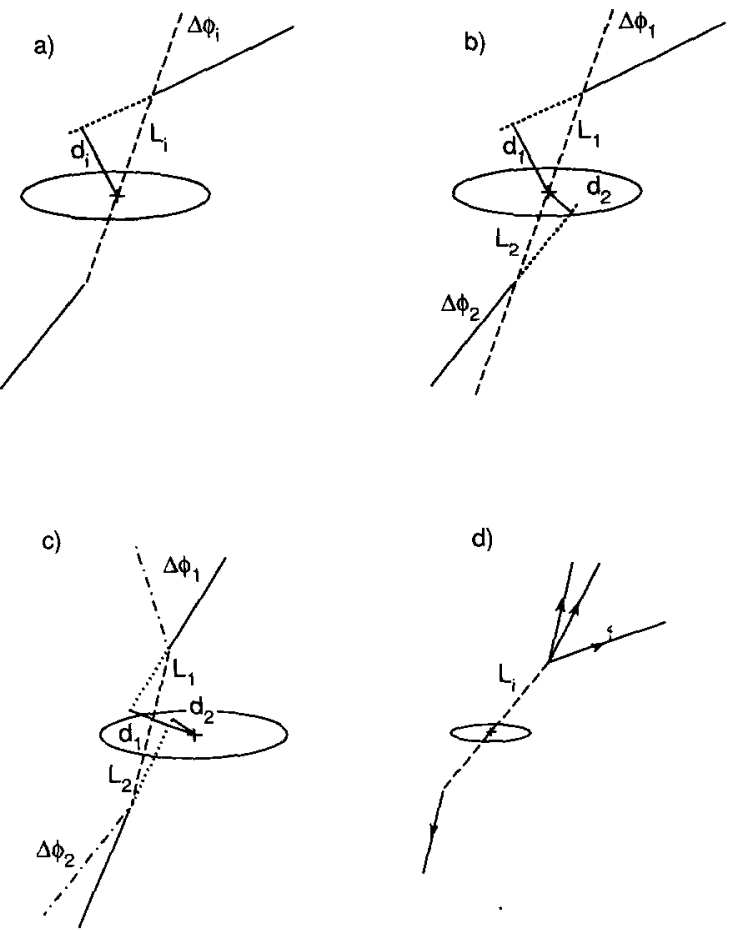

Fig. 1. Schematic diagrams showing the quantities used to extract the tau lifetime in (a) the impact parameter measurement, (b) the decay-angle correlation measurement, (c) the miss distance measurement and (d) the vertex measurement.

(v) the particle transverse momentum, $p_{t_{i}}$, was greater than $1 \mathrm{GeV} / c$;

(vi) there be at most one VD layer with an unassociated hit within $7.5^{\circ}$ of the track in $\phi$. This removed a small number of events with conversions, delta-rays and three prong decays where the other two tracks were unassociated in the VD;

(vii) if the track had hits in only two layers of the VD, there should not be any other hit within $400 \mu \mathrm{m}$, in order to reduce the mis-association of hits to the track;

The geometrically signed impact parameter, $d_{i}$, is the distance of closest approach of the extrapolated track to the assumed production point, the centre of the interaction region, in the $r \phi$ plane;

$d_{i}=L_{i} \sin \theta_{\tau_{i}} \sin \left(\phi_{i}-\phi_{\tau_{i}}\right)$,

where $L_{i}$ is the decay length, $\phi_{\tau_{i}}$ and $\theta_{\tau_{i}}$ are the azimuthal direction and polar angle of the decaying tau while $\phi_{i}$ is the azimuthal angle of the tau decay product (see fig. 1a ). Experimentally, the sign of the geometric impact parameter is defined as the sign of the $z$ component of the vector cross-product of the projections on the $r \phi$ plane of the track vector at the point of closest approach and the vector from the centre of the interaction region to the point of closest approach.

\subsection{The impact parameter method}

For tau decays producing a single charged particle, the lifetime signed impact parameter was used which differs from the geometric impact parameter only in its sign. The sign is positive if the extrapolated track intersects the tau direction before reaching the point of closest approach and negative otherwise. If the geometry of the production and decay could be reconstructed perfectly, the lifetime signed impact parameter would always be positive. Because of resolution effects and uncertainties in the tau direction it can be negative. However the distribution of lifetime signed impact parameters remains sensitive to the tau lifetime.

The tau production point was taken as the centre of the interaction region. The decay particle in the opposite hemisphere was used only as an estimate of the tau direction for the sign of the impact parameter. Monte Carlo simulation showed that the difference between this particle direction and the tau direction was centred on zero with a width of about $2^{\circ}$.

The lifetime was extracted from the lifetime signed impact parameter distribution using a maximum likelihood fit. The lifetime signed impact parameter probability distribution was determined as a function of the tau lifetime as follows: impact parameter distributions, for different lifetimes, were generated using Monte Carlo events in which the effects due to tau decay kinematics and experimental cuts for tau selection were included, but assuming perfect detector resolution and a point interaction region. In what follows this simulated impact parameter distribution is referred to as the physics function. In order to account for the smearing due to the beam size and the track extrapolation resolution, this impact parameter distribution was convoluted with a resolution function obtained from the geometric impact parameter distribution of the $\mu^{+} \mu^{-}$and $e^{+} e^{-}$events. 
The effect of multiple scattering was accounted for by smearing the physics function decay by decay as described in eq. (2). The uncertainty in the lifetime due to uncertainty in the multiple scattering term was found to be negligible. A contribution to the physics function was included to account for the small number of elastic hadronic interactions expected in the beam pipe and layers of the VD.

The background contamination of the sample was determined from Monte Carlo simulation to be (1.6士 $0.4) \%$, due to $e^{+} e^{-}, \mu^{+} \mu^{-}$and two photon events. A background contribution represented by the resolution function, suitably normalised and centred on zero, was included in the probability distribution. The final data sample comprised 6117 tau decays.

The log-likelihood was formed to determine the best fit lifetime and its uncertainty. To obtain the optimal statistical uncertainty, the data were grouped into six bins as a function of the apparent beam profile, which was symmetric under reflections in the $x$ and $y$ axes. Thus each bin contained data from the four 15 degree wide sectors in $\phi$, one in each of the four quadrants, which mapped onto one another under reflection in the $x$ and $y$ axes. These should have seen the same beam profile. In each bin, a combined maximum likelihood fit of the lifetime and the resolution function was made. The resolution function was parameterised as a gaussian. The lifetime was taken as the weighted mean of the results of the six binned fits.

The lifetime was found to be $303 \pm 10$ fs using a resolution function made up of $20 \%$ electrons and $80 \%$ muons. Muons were taken to represent the resolution expected for all other tau decay products. Fig. 2 shows the measured impact parameter distribution with the probability distribution calculated for this lifetime superimposed. The result obtained using only $\mu^{+} \mu^{-}$for the resolution function was $304 \mathrm{fs}$ and that obtained using only $e^{+} e^{-}$was $301 \mathrm{fs}$, showing no evidence of systematic effects because of differences in tracking due to particle type.

The analysis procedure was tested for bias using a sample of Monte Carlo events with full detector simulation showing that the systematic effects in the analysis method were less than $3 \mathrm{fs}$. Other systematic uncertainties arose from: the uncertainty in the radial alignment of the VD ( $1 \mathrm{fs}$ ); the uncertainty in the parameterisation of the resolution function ( $3 \mathrm{fs}$ ); the uncertainty on the contamination in the sample of taus

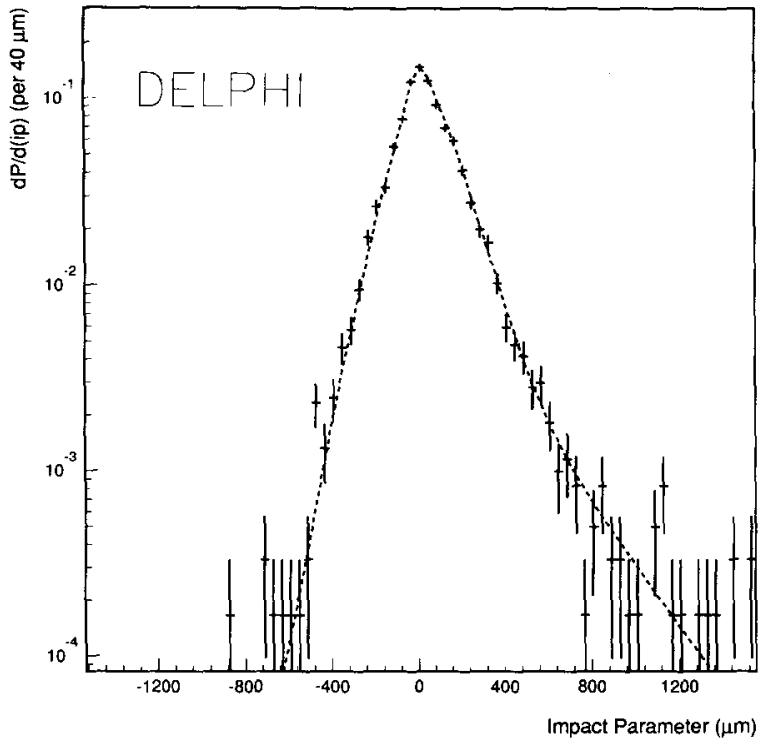

Fig. 2. The observed lifetime signed impact parameter distribution for taus. The crosses are the data points while the curve shows the probability distribution for the fit value of the tau lifetime.

( $1 \mathrm{fs}$ ); the effect of hadronic scattering ( $2 \mathrm{fs}$ ); uncertainties in the tau branching fractions ( $1 \mathrm{fs}$ ). Added in quadrature, these gave a total systematic uncertainty of $5 \mathrm{fs}$. As a further check on the consistency of the data, the lifetime was calculated for positively and negatively charged decay particles, for various cutoff values of $p_{\mathrm{t}}$, for positive and negative $z$ and for different impact parameter fit ranges. All values of the lifetime obtained were consistent. The final result from the impact parameter method was

$\tau_{\tau}=303 \pm 10$ (stat.) \pm 5 (syst.) fs.

\subsection{The decay-angle correlation method}

The impact parameter of a tau decay product is generated both by the flight distance of the decaying tau and the angle the decay particle makes with the original tau direction. This can be exploited to determine the tau lifetime by correlating the impact parameters and the difference in azimuthal angles of the tau decay products [7].

Taking $d_{i}$ from eq. (3), the geometrically signed impact parameter of each decay product to the inter- 
action point, we can form the impact parameter difference

$$
\begin{gathered}
d_{1}-d_{2}=L_{1} \sin \theta_{\tau_{1}} \sin \left(\phi_{1}-\phi_{\tau_{1}}\right) \\
-L_{2} \sin \theta_{\tau_{2}} \sin \left(\phi_{2}-\phi_{\tau_{2}}\right) .
\end{gathered}
$$

Neglecting photon radiation the taus are produced back to back, giving $\phi_{\tau_{1}}-\phi_{\tau_{2}}=\pi$ and $\sin \theta_{\tau_{1}}=$ $\sin \theta_{\tau_{2}} \equiv \sin \theta$ (see fig. lb ). Averaging over decay lengths $\left(\left\langle L_{1}\right\rangle=\left\langle L_{2}\right\rangle \equiv\langle L\rangle\right)$ and approximating $\sin \left(\phi_{1}-\phi_{\tau_{1}}\right) \approx \phi_{1}-\phi_{\tau_{1}}$, since the tau decay product follows the tau direction to about $2^{\circ}$, gives

$$
\begin{aligned}
& \left\langle d_{1}-d_{2}\right\rangle=\langle L\rangle \sin \theta\left(\phi_{1}-\phi_{2}+\pi\right) \\
& \quad=\langle L\rangle \sin \theta \Delta \phi .
\end{aligned}
$$

The average decay length, $\langle L\rangle$, is $\beta \gamma c \tau_{\tau}$. Thus the mean impact parameter difference, $\left\langle d_{1}-d_{2}\right\rangle$ is proportional to the projected acoplanarity $(\sin \theta \Delta \phi)$ with a proportionality constant, $\langle L\rangle$, which is related to the tau lifetime.

The variables $d_{1}, d_{2}, \Delta \phi$ and $\theta$ were measured on an event by event basis to extract the correlation (eq. (5) ), $\theta$ being estimated from the direction of the thrust axis. Monte Carlo simulation showed that the difference between the thrust axis and the tau direction was centred on zero with a width of about $1^{\circ}$. This method had a reduced dependence of the lifetime on the unmeasured tau decay angles. While $\phi_{i}-\phi_{\tau_{i}}$ could not be determined event by event the difference, $\Delta \phi$, was measured with a precision of $0.5 \mathrm{mrad}$. Another advantage of this method is that backgrounds such as $\mu^{+} \mu^{-}, e^{+} e^{-}$events tend to have small $\Delta \phi$ and hence had a reduced effect on the correlation determination. The prime drawback of this method was that $d_{1}-d_{2}$ was doubly smeared by the lack of knowledge of the tau pair production point inside the interaction region. Moreover other backgrounds such as two photon events or radiative $\mu^{+} \mu^{-}$and $e^{+} e^{-}$events had $\left\langle d_{1}-d_{2}\right\rangle \approx 0$ independent of the projected acoplanarity. This gave a bias towards smaller lifetimes.

This method did not require as precise a knowledge of the track extrapolation resolution as the other methods. Thus, for this analysis, both tracks were required to satisfy only criteria $2,3,5$ and 7 from above, in order to maximise the data sample. The presence of a

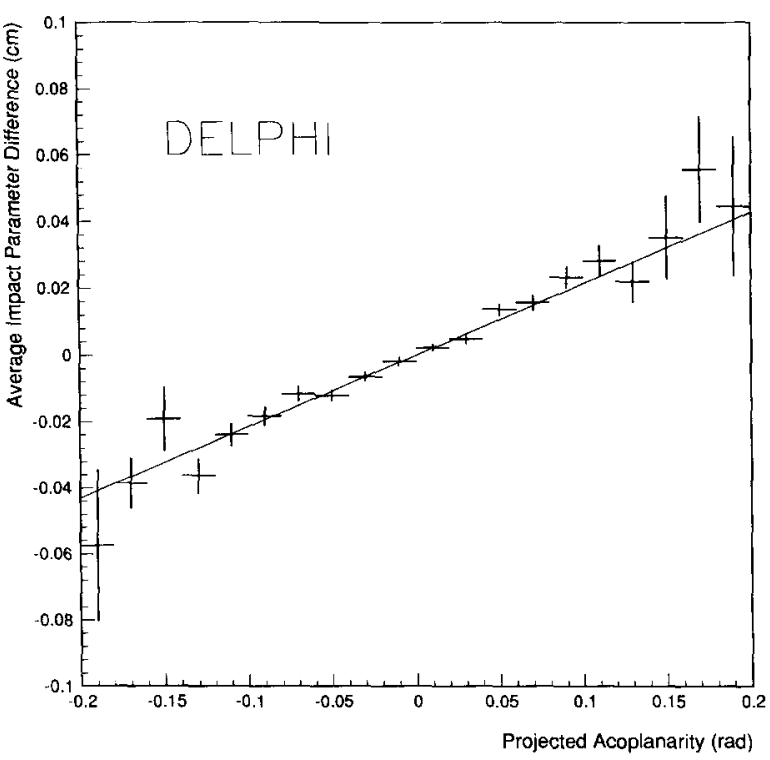

Fig. 3. The average impact parameter difference, $\left\langle d_{1}-d_{2}\right\rangle$, versus the projected acoplanarity, $\sin \theta \Delta \phi$. The slope of the fit line is $(2.15 \pm 0.08$ (stat.) ) $\mathrm{mm}$ from which the tau lifetime has been extracted.

photon in $\tau^{+} \tau^{-} \gamma$ events can increase the acoplanarity, independent of the impact parameter difference, biasing the measured lifetime. These events were removed by discarding events with a photon of $E>1 \mathrm{GeV}$ creating an invariant mass with the closest charged particle of more than $2 \mathrm{GeV} / \mathrm{c}^{2}$. The two photon background was reduced by requiring that the two particles have opposite charge. This left 2880 events with $|\sin \theta \Delta \phi|<0.2$ radians used for the lifetime determination.

A straight line was fit to determine the correlation between acoplanarity and impact parameter difference, weighting each event according to the uncertainty, $\sigma$, on $d_{1}-d_{2}$. These weights $\left(1 / \sigma^{2}\right)$ included the track extrapolation uncertainty, interaction region size, and the physical width of the exponential tau decay distribution predicted by Monte Carlo simulation. The slope of the line was used to determine the lifetime as shown in eq. (5). The fit was iterative, removing events with poor significance (residual $/ \sigma$ ). Fig. 3 shows the mean $\left\langle d_{1}-d_{2}\right\rangle$ for slices of $\sin \theta \Delta \phi$ after removing $0.8 \%$ of the events with the largest residual $/ \sigma$. The fit slope was

$\langle L\rangle=2.148 \pm 0.081$ (stat.) \pm 0.019 (syst.) $\mathrm{mm}$. 
The systematic uncertainty on the slope comes from variations in the fit range and the determination of the weights used. The iterative procedure removed poorly reconstructed events as well as a few very acoplanar tau decays. The removal of these latter events resulted in a bias of $+0.138 \pm 0.031 \mathrm{~mm}$ on the slope - a correction that was included when interpreting the slope as a lifetime. The uncertainty on this bias is statistical. With a larger data sample fluctuations in the number of well measured tau decays removed would be smaller and the bias could be better determined.

As mentioned above, most backgrounds $\left(e^{+} e^{-}\right.$, $\mu^{+} \mu^{-}$and cosmic rays which amount to $(1.2 \pm 0.4) \%$ of the final sample) do not affect the measured slope, but a $+1.2 \pm 3.5$ fs correction was made for the $(0.18 \pm 0.07) \%$ remaining radiative tau decays and two photon events in the sample. The large uncertainty on this correction came from the small number of two photon background events which remained in the sample. Poisson fluctuations in this number of events were included in the estimate of the systematic uncertainty coming from the influence of this background. Other biases were negligible.

Additional systematic uncertainties arose from the VD alignment ( $2 \mathrm{fs}$ ), resolution function determination ( $1 \mathrm{fs}$ ), event selection ( $2 \mathrm{fs})$ and uncertainties on the effect of the fit procedure ( $2 \mathrm{fs}$ ).

The slope was interpreted, including the biases mentioned above, as a tau lifetime of

$\tau_{\tau}=300 \pm 12$ (stat.) \pm 6 (syst.) fs,

where the statistical uncertainties on the measured slope and bias were combined to give the overall statistical uncertainty.

\subsection{The miss distance method}

The miss distance method used both decay particles in a 1-1 topology event, similar to the decay-angle correlation method. The definition and signing of the impact parameters here were also the same.

In the impact parameter and decay angle correlation methods the knowledge of the tau pair production point was limited by the size of the interaction region, dimensions of which are much larger than the resolution on extrapolations to the interaction region. To overcome this limitation the two impact parameters in a $\tau^{+} \tau^{-}$event were summed so that the depen- dence on the interaction region cancelled to first order (see fig. 1c ). The resulting quantity $d_{\text {miss, }}$, called the miss distance, was given by

$d_{\text {miss }}=d_{1}+d_{2}$

where $d_{1}$ and $d_{2}$ were defined in eq. (3).

The lifetime was estimated by fitting simulated miss distance distributions to the data with a maximum likelihood technique. The simulated distributions were made from the convolution of a physics function created for perfect detector resolution including multiple scattering effects and a resolution function measured from $\mu^{+} \mu^{-}$and $e^{+} e^{-}$events.

Both particles in the event were required to satisfy the criteria described in section 3.1. This gave a final sample of 2369 events which was used to extract the lifetime with the miss distance method.

The resolution function, $R(x)$, was derived from the miss distance of $\mu^{+} \mu^{-}$events. This was parameterised as a sum of two gaussian distributions of the form

$$
\begin{aligned}
& R(x)=(1-f) \exp \left(-x^{2} / 2 \sigma_{1}^{2}\right) \\
& \quad+f \exp \left(-x^{2} / 2 \sigma_{2}^{2}\right),
\end{aligned}
$$

with the values $\sigma_{1}=30.8 \mu \mathrm{m}, \sigma_{2}=59.3 \mu \mathrm{m}$ and $f=0.16$. Studies showed that the presence of two gaussians was attributable to classes of tracks with different extrapolation uncertainties, arising from the number of VD hits associated with different tracks and the radii at which these hits were measured. With this resolution function a value of the lifetime of $294 \pm$ 9 fs was determined. Fig. 4 shows the tau miss distance distribution with this fit superimposed. To estimate the effect of biases in the event selection a sample of Monte Carlo events, with full detector simulation, was selected and fitted in the same way as the data. This yielded a lifetime of $302 \pm 2 \mathrm{fs}$, in good agreement with the input lifetime of $300 \mathrm{fs}$. The value of $2 \mathrm{fs}$ was taken as an estimate of the possible bias in the method.

An estimate of the systematic uncertainty arising from the knowledge of the resolution function was made by using $e^{+} e^{-}$events, by using single gaussian fits to $\mu^{+} \mu^{-}$and $e^{+} e^{-}$events, and by separating the data into classes with different VD layer combinations. From these studies a systematic uncertainty of 


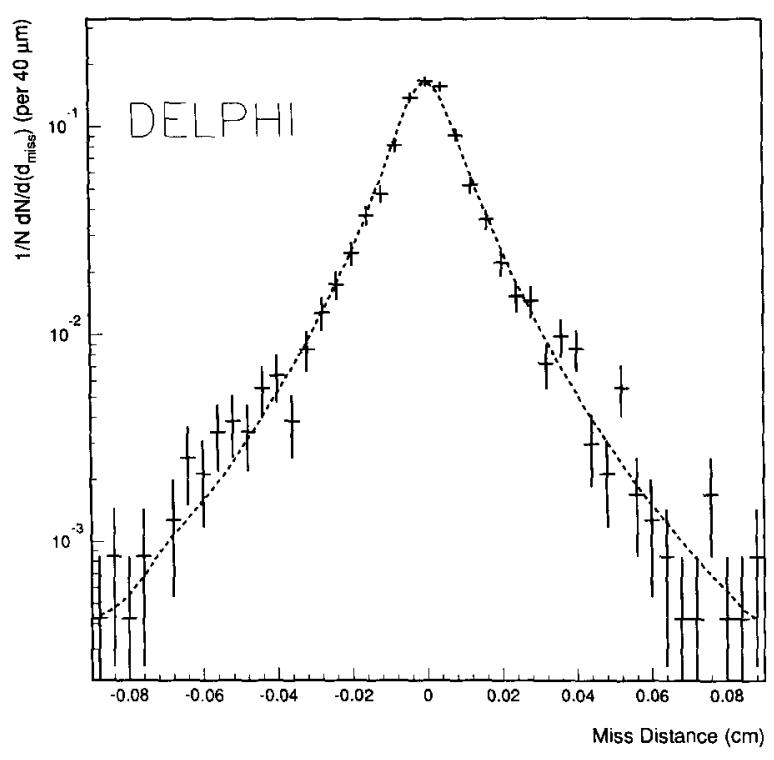

Fig. 4. The tau miss distance distribution. The crosses are the data points while the dashed line is the best fit from which the tau lifetime was determined.

3 fs was assigned due to resolution uncertainties. The statistical uncertainties on the parameters in eq. (7) are small and necessitate no additional contribution to the lifetime systematic. The fitted lifetime with the two gaussian resolution function in $e^{+} e^{-}$events was $3 \mathrm{fs}$ lower than for $\mu^{+} \mu^{-}$events. A bias of $+0.6 \pm 0.6 \mathrm{fs}$ has already been included to account for the electrons making up about $20 \%$ of one prong $\tau$ decays.

The background from $e^{+} e^{-}, \mu^{+} \mu^{-}$and two photon events of $(1.0 \pm 0.3) \%$ was accounted for by adding a suitably normalised delta function to the physics function at zero miss distance. This gave a 1 fs systematic uncertainty on the lifetime. Residual alignment uncertainties and knowledge of multiple scattering were combined to give an additional $1 \mathrm{fs}$ contribution to the systematic uncertainty on the lifetime.

The uncertainty in the mean tau longitudinal polarisation was estimated by varying $\sin ^{2} \theta_{w}$, while correlations between the transverse spin components were estimated using KORALB [8]. These uncertainties taken together with possible variations in the tau branching ratios contributed another 1 fs to the systematic uncertainty on the lifetime.

The range over which the fit was performed was chosen after study of data and fully simulated Monte
Carlo events to be $\pm 0.9 \mathrm{~mm}$. This minimised the effects of tails due to elastic hadronic scattering while maintaining sensitivity to the lifetime. This choice corresponded to a trim of $0.8 \%$ of the data, consistent with the amount expected from the hadronic interaction probability in the beampipe and VD. Reducing the range further did not produce any significant deviation in the estimated lifetime beyond that expected from statistical fluctuations.

No significant dependence of the measured lifetime on the selection cuts was found. The different systematic uncertainties were added together in quadrature, giving a tau lepton lifetime of

$\tau_{\tau}=294 \pm 9$ (stat.) \pm 4 (syst.) fs.

\section{The vertex method}

In the sample of tau decays to three charged particles, the decay vertex was reconstructed allowing a direct measurement of the tau flight distance (see fig. 1d) and thence the lifetime. The three charged tracks were required to have an invariant mass of less than $2 \mathrm{GeV} / c^{2}$ and the other tau was required to decay to a single charged particle in order to reduce the hadronic background. Monte Carlo studies showed that the remaining background was $(1.0 \pm 0.3) \%$. A total of 2159 events with three prong momentum sum having a polar angle between $20^{\circ}$ and $160^{\circ}$ was selected for the analysis.

In order to achieve the necessary precision on the vertex determination, VD hits had first to be associated to the external tracks, which were composed of track elements from the TPC and the ID. The ID was included here to remove hit association ambiguities among the three tracks present in the VD in each decay. The tracks were extrapolated to the VD and all combinations of hits occurring within a road of suitable dimensions were considered. The width of this road was set to three times the calculated extrapolation uncertainty. A total of 1207 decays had at least two VD hits on each of the three tracks and hence were retained for further analysis.

For each combination of hits a circle fit was made in the transverse plane, accounting for multiple scattering. The $\chi_{\text {track }}^{2}$ probability distribution was flat showing that the point resolution in the various detectors 
and the multiple scattering were correctly determined. A cut of $\chi_{\text {track }}^{2}$ probability less than 0.01 removed a small number of incorrect hit associations. In general only one good combination of VD hits existed for each of the three tracks. In the case where more than one combination existed all were considered and the possible ambiguity was solved at the next stage. A total of 1163 vertices survived to this stage in the analysis.

In order to reduce false associations, to solve ambiguities in the track fits and to remove background from photon conversions, the constraint that the three tracks produce a good vertex was imposed. The decay vertex position $(x, y)$ was estimated by minimising the function

$\chi_{\text {vertex }}^{2}(x, y)=\sum_{j}\left(\frac{d_{j}}{\sigma_{j}}\right)^{2}$,

where $d_{j}$ is the distance of closest approach to the vertex $(x, y)$ in the $r \phi$ plane of particle $j(j=1,2,3)$, and $\sigma_{j}$ is the extrapolation uncertainty at the decay vertex as calculated from the track fit.

The $\chi_{\text {vertex }}^{2}$ probability distribution for the reconstructed decay vertex was flat except for a large peak towards zero corresponding to incorrect associations and background events. Again a cut on $\chi_{\text {vertex }}^{2}$ probability of less than 0.01 was made and all the remaining possible vertices for a given event were considered. In general there was only one, but where two or more existed the event was rejected unless the decay vertex error ellipses overlapped at the two standard deviation level in which case the vertex with the smallest uncertainty was retained for further analysis. This left a final data sample of 838 vertices.

To determine the projected decay length, $l_{i}$, the production point was taken to be the centre of the interaction region. The laboratory decay length $L_{i}$ was calculated from

$L_{i}=\frac{l_{i}}{\sin \theta_{i}}$

where $\theta_{i}$ is the polar angle of the tau which was approximated as that of the thrust axis of the three charged particles in the decay. The distribution of $L_{i}$ is shown in fig. 5 . The uncertainty on this, $\sigma_{L_{i}}$, was calculated from the covariance matrix for the position of the decay point and from the uncertainty in the actual production point. The typical uncertainty on the decay

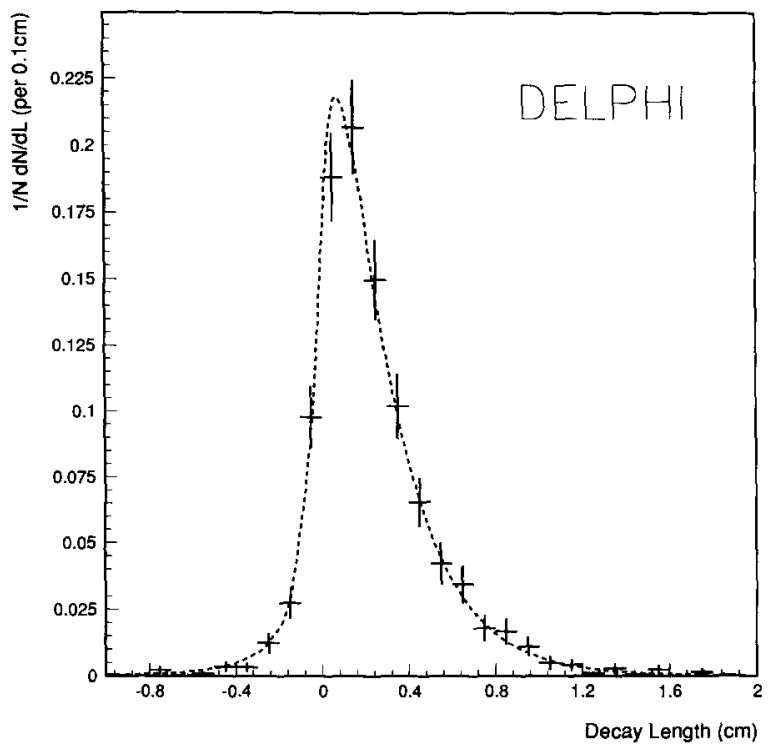

Fig. 5. The observed decay length distribution for taus using the vertex method. The crosses are the data and the curve represents the best fit from which the tau lifetime was determined.

length was $750 \mu \mathrm{m}$, which is about one third the size of the mean decay length.

The decay time in the rest frame of the tau $T_{i}$ is given by

$T_{i}=\frac{L_{i}}{\beta \gamma \mathrm{c}}$,

where $\beta \gamma=p_{\tau} / m_{\tau}$ with $p_{\tau}$ determined from the beam energy taking account of radiative corrections. The lifetime was extracted from the distribution of decay times using the maximum likelihood method. For each event, the probability of the event having a decay time $T_{i}$ was calculated as a function of the lifetime using an exponential lifetime distribution convoluted with a resolution function. For the ideal case, including detector resolutions and multiple scattering, the resolution function would be a gaussian of width $\sigma_{T_{i}}$ (derived from $\sigma_{L_{i}}$ using eq. (10)). Monte Carlo studies indicated that this had to be modified to take into account the effect of hadronic scatters, particularly in the inner wall of the TPC. The parameterisation obtained for the resolution function was 


$$
\begin{aligned}
& R(x)=0.940 G\left(x ; \sigma_{T_{i}}\right)+0.058 G\left(x-0.4 \sigma_{T_{i}} ; 3 \sigma_{T_{i}}\right) \\
& \quad+0.002
\end{aligned}
$$

where $G(x ; \sigma)$ was a gaussian of width $\sigma$ centred on zero and with unit area. The first term corresponded to the ideal case while the second and third terms account for the detector effects discussed above. No cut was found which enabled such mismeasured decays to be removed from the event sample.

The procedure was tested by analysing simulated events with five known lifetimes between $100 \mathrm{fs}$ and $500 \mathrm{fs}$. The results showed that the systematic effects associated with the analysis technique were less than $2 \mathrm{fs}$. Both the systematic uncertainty arising from ambiguities in the association of the VD hits and that arising from uncertainties on the tau direction were negligible. Uncertainties in the effect of initial and final state radiation ( $1 \mathrm{fs}$ ), and in the radial alignment of the VD ( $2 \mathrm{fs}$ ) have also to be included. The effect of hard hadronic scatters on the resolution function parameterised in eq. (11) gave rise to a $3 \mathrm{fs}$ contribution to the systematic uncertainty on this method. The background from hadronic decays of the $Z$ necessitated a 3 fs upwards correction to the lifetime (included in the fit above) and resulted in an additional 2 fs systematic uncertainty. By adding all contributions in quadrature the total systematic uncertainty was estimated to be $5 \mathrm{fs}$.

The final result of the vertex method was

$\tau_{\tau}=298 \pm 13$ (stat.) \pm 5 (syst.) fs.

\section{Summary and conclusions}

The lifetime of the tau has been measured in four ways. The first three methods used data samples with a very large overlap but were not completely statistically correlated since they exploited different event properties. Furthermore, the systematic uncertainties involved were different, providing an important cross-check. A 50\% correlation between the impact parameter and decay angle correlation methods was determined by comparing the fit lifetime results from Monte Carlo $Z \rightarrow \tau^{+} \tau^{-}$samples. Similarly $30 \%$ and $20 \%$ correlations were determined between the impact parameter - miss distance measurements
Table 1

Summary of the systematic uncertainties on each of the four measurements in fs. Grouped under Physics are contributions from the tau branching ratios, radiative corrections and tau polarisation.

\begin{tabular}{lllll}
\hline $\begin{array}{l}\text { Origin of } \tau_{\tau} \\
\text { systematic }(\mathrm{fs})\end{array}$ & Impact & $\begin{array}{l}\text { Decay- } \\
\text { angle }\end{array}$ & $\begin{array}{l}\text { Miss } \\
\text { distance }\end{array}$ & Vertex \\
\hline alignment & 1 & 2 & 1 & 2 \\
resolution function & 3 & 1 & 3 & 3 \\
background & 1 & 3 & 1 & 2 \\
fit method & 3 & 3 & 2 & 2 \\
physics & 2 & 4 & 1 & 1 \\
total & 5 & 6 & 4 & 5 \\
\hline
\end{tabular}

and the decay angle correlation - miss distance measurements respectively. As the three measurements agreed well, they were combined, accounting for the correlations, to give a lifetime for the one prong decays of $298 \pm 7$ (stat.) \pm 4 (syst.) fs, leaving two statistically independent measurements, those from the one prong decays and the three prong decays. Of the systematic uncertainties only those arising from the VD alignment were common. A summary of all the systematic uncertainties is given in table 1 .

Combining the one and three prong lifetime measurements by weighting them with the reciprocal of the quadratic sum of the statistical and independent systematic uncertainties and retaining the common systematic uncertainty unaltered, a tau lifetime of

$\tau_{\tau}=298 \pm 7 \mathrm{fs}$,

was obtained. This result agrees with the value of $289 \pm 3$ fs predicted by eq. (1) using $B R(\tau \rightarrow e \nu \nu)=$ $(17.69 \pm 0.19) \%[9]$ and $m_{\tau}=1776.9 \pm 0.5 \mathrm{MeV} / c^{2}$ [10]. Alternatively the measured lifetime may be used to determine the relative strengths of the Fermi coupling constants $\left(G_{\tau} / G_{\mu}\right)$. This ratio was found to be $0.985 \pm 0.013$, consistent with lepton universality.

The measurement presented here agrees well with other recent measurements $[7,11]$. Although systematic uncertainties are important, future measurements will benefit from the increased statistics expected from ongoing LEP running. This will then leave us in a position to make very precise $(<1 \%)$ tests of lepton universality. 


\section{Acknowledgement}

We are greatly indebted to our technical collaborators and to the funding agencies for their support in building and operating the DELPHI detector, and to the members of the CERN-SL Division for the excellent performance of the LEP collider.

\section{References}

[1] Y.S. Tsai, Phys. Rev. D 4 (1971) 2821, D 13 (1976) 771 ;

H.B. Thacker and J.J. Sakurai, Phys. Lett. B 36 (1971) 103.

[2] DELPHI Collab., P. Abreu et al., Nucl. Phys. B 367 (1991) 511.

[3] S. Jadach and Z. Was, Comput. Phys. Commun. 36 (1985) 191;

S. Jadach et al., CERN 89-08, Vol. 3 (1989) 67.

[4] DELPHI Collab., P. Aarnio et al., Nucl. Instrum. Methods A303 (1991) 233.
[5] N. Bingefors et al., preprint CERN-PPE 92/173, submitted to Nucl. Instrum. Methods.

[6] DELPHI Collab., P. Aarnio et al., Phys. Lett. B 260 (1991) 240.

[7] ALEPH Collab., D. Decamp et al., Phys.Lett. B 279 (1992) 411.

[8] S. Jadach and Z. Was, Proc. XXVI Intern. Conf. on High energy physics (Dallas, USA, 1992).

[9] Particle Data Group, K. Hikasa et al., Review of particle properties, Phys. Rev. D 45, part 2 (1992); CLEO Collab., Phys. Rev. Lett. 69 (1992) 3610.

[10] BES Collab., J.Z. Bai et al., Phys. Rev. Lett. 69 (1992) 3021.

[11] L3 Collab., B. Adeva et al., Phys. Lett. B 265 (1991) 451;

OPAL Collab., P.D. Acton et al., Phys. Lett. B 273 (1991) 355;

DELPHI Collab., P. Abreu et al., Phys. Lett. B 267 (1991) 422. 